\title{
Controlled optimization model for traffic zones during road repair periods
}

\author{
Elizaveta Kondrashova ${ }^{1 *}$ \\ ${ }^{1}$ Moscow State University of Civil Engineering, Yaroslavskoe h. 26, Moscow, 129327, Russia
}

\begin{abstract}
The aim of the research is construct a model that allows to make the best decisions while carrying out repairs on the road. These decisions can be made taking into account both the interests of the participants of the traffic and taking into account the costs spent on repair. This paper presents optimization model for traffic zones using queueing controlled models in searching for an optimal management strategy. This optimal strategy is based on the theory of controlled semi-Markov processes. As a result we have controlled model for use during road repair periods. The model can be complicated and varied depending on the conditions.
\end{abstract}

\section{Introduction}

Any road repairs cause difficulties in road traffic. The question arises as to how to optimize road traffic in case of road repairs. Recently the transport flow optimization problems become more relevant. The problem of transport traffic: multi-hour traffic jams, overload of roads, increase of road traffic accidents, all it leads to the fact of traffic's becoming more difficult. It leads to a non-economy of personal and work time of vehicular traffic participants. Recently, more and more authors are turning to both new methods of research and solving optimization problems of transport management, as well as modifying existing methods, which gives quite successful results [1-7].

Queueing models allow to investigate the efficiency of the system functioning. Dependencies of system functioning efficiency on its structure and initial characteristics are established and optimization problem is solved.

Erlang dealt with the problems of mass service in the early 20th century, when the main problem in the field of telephony was to organize a telephone message to provide guaranteed service. Despite the fact that quite a long time has passed, similar problems arise at present. In order to meet the requirements of the system, it is not possible for economic reasons to increase resources without limits, such as the number of serving channels, the number of places in the queue, the capacity, etc.

Thus, one of the main problems is identified, in which the methods of queueing theory are used, namely, the search for control, which takes into account both the improvement of the quality of service and the permissible costs associated with the improvement of the system operation. Note that with the help of theory a large number of analytical results

\footnotetext{
*Corresponding author: elizavetakondr@gmail.com
} 
characterizing different qualities of systems were obtained.

Some part of the methods using markov processes for traffic regulation was investigated in some research paper [8-13]. It is proposed to use the controlled semi-markov queuing model to optimize road repairs.

\section{The problem of receiving a request to enter restricted traffic zones during road repair periods}

We review a road zone with two lanes in different directions (one lane has a specified direction, the other is used for oncoming traffic). Repairs on the road surface are underway. Repairs can be performed at a fixed point on the roadway, but it is not possible to pass this point without permission from the repair team/police. We suppose that there is an option to arrange left-hand and right-hand regulation of the traffic for each lane. A group of machines will therefore accumulate at a certain fixed point in front of the repairing vehicles on the road. We will assume that there is some box of requests (units of vehicles intending to pass). At certain moments, the repairing vehicles "stops" the entire cycle of work underway (for example, they start partially functioning, allowing vehicles to pass) and then "allows" other vehicles to pass. Incoming requests are made by road vehicles intending to pass the point. Passing is therefore only possible at the time permitted.

Therefore we can say that we are reviewing two types of request determined according to number of lanes. The number of requests of a certain type that the system accepts for "maintenance" is a managed object. Moments at which vehicles are given "permission" to enter are chosen in order to optimize the zone's traffic situation (or in order to optimize the operation of repairing equipment); accordingly, these moments can be considered to be a controlling action regarding the functioning of the road in general. The repairing vehicles under the service channel should allow ordinary traffic to pass through at "permission" moments.

Let us consider vehicles (queries) intending to carry out entrance from CBSMAP-flow [14-16]. Two types of queries are considered in the given model: motor transport units intending to carry out entry in the direction $i=1$ (type 1), and motor transport units intending to carry out entry in the direction $i=2$ (type 2). Note that it is very reasonable to change the characteristics of arrival flows in various models to optimize their functioning. The control is based on the theory of controlled semi-Markov processes for system optimization [14-16]. Control can be carried out using batch arrival "moments", the type of the queries, and the quantity of queries within the batch.

\section{Algorithmization and results}

The Controlled Batch semi-Markov Arrival Process flow (CBSMAP-flow) is a generalization of the Batch Markov Arrival Process (BMAP) flow model [17]. Define CBSMAP-flow $[15,16]$. After holding in that state comes to an end, the controlled semiMarkov process jumps to the other state, and the batch of queries of CBSMAP-flow are generated. All major characteristics for the CBSMAP-model were obtained from previous papers [14-16].

An incoming stream of conditions does not lack an aftereffect (Markov property) at arbitrary moments in time. Managed input is defined by a managed semi-Markov process $(t)=\{\xi(t), u(t)\}$, whereby the first component takes values from a finite set, $\xi(t) \in E=$ $\{1,2, \ldots, N\}$. The designation of the CBSMAP stream was also introduced for this incoming stream. Note that this incoming stream of conditions can be considered to be a generalization of the BMAP stream previously introduced by [17]. The designation entered 
is therefore interpreted as controlled batch semi-Markov arrival process (CBSMAP). The receipt of applications can occur only at the moment of change among a finite set of states of a certain semi-Markov process that have continuous time.

The states of the queuing system $(i, \vec{l})=\left(i, l_{1}, l_{2}, \ldots, l_{N}\right)$ are determined by the vector where $i$ - is the state of the input stream (the group of conditions of the $i$ type arrives at the Markov moment); $l_{k}-$ is the number of conditions in the $k$-type subsystem. For the state space of a discrete component $\times E_{1} \times \ldots \times E_{N}=\tilde{E}$.

In the adopted notation of the semi-Markov process, states can be denoted by $(i, \vec{l})$ and written as $(i, \vec{l}) \in \tilde{E}$. The Markov control strategy $\vec{G}=\left\{G_{(i, \vec{m})}(u),(i, \vec{m}) \in \tilde{E}\right\}$, only depends on the current state of the controlled process, and a set of probability measures are defined for each state, on $\sigma$ - the algebra of the solution set subsets is $U_{(i, \vec{l})}$.

The functional of the specific accumulated income of a $k$-type system [16] is valid:

$$
S^{(k)}=\frac{\sum_{(i, \bar{l}) \in \widetilde{E}^{S}}{ }_{(i, \bar{l})}^{(k)} \pi_{(i, \bar{l})}^{(k)}}{\sum_{(i, \bar{l}) \in \widetilde{E}} m_{(i, \bar{l})}^{(k)} \pi_{(i, \bar{l})}^{(k)}}
$$

and for the system in sum: $S=\sum_{k=1}^{N} S^{(k)}$, where $m_{(i, \vec{m})}^{(k)}$ is the mathematical expectation of time of continuous process $\xi(t)$ in state $(i, \vec{m})$; and $s_{(i, \vec{m})}^{(k)}$ is the mathematical expectation of the accumulated income during the operation of the queueing model $(k)$ during the continuous process $\xi(t)$ in state $(i, \vec{m})$.

As shown earlier in [16], the functionals $S, S^{(k)}$ are linear-fractional with respect to distributions that determine the accumulation structure. Consequently, the maximum of a linear-fractional functional can be found on the set of degenerate distributions. To optimize the income functional, we use earlier expressions obtained from existing researches [14-16] for each k queuing subsystem: $p_{(i, \vec{m})(j, \vec{l})}^{(k)}$ is the transition probabilities of the embedded Markov chain; $m_{(i, \vec{m})}^{(k)}$ is the mathematical expectation of time of the continuous process $\xi(t)$ in a state $(i, \vec{m})$; and $s_{(i, \vec{m})}^{(k)}$ is the mathematical expectation of the accumulated income during the operation of the queueing system $(k)$ during the continuous process $\xi(t)$ in state $(i, \vec{m})$.

To solve this problem, it is necessary to define the functions - conditional mathematical expectation of the accumulated income in the $Q S(k)$ - provided that the process is in $\operatorname{state}(i, \vec{m})$, and that, after $t$ time, it passes into the $(i, \vec{l})$ state and a decision $u$ is made.

The conditional mathematical expectation of the accumulated income $R_{(i, \vec{m})(j, \vec{l})}^{(k)}(x, u)$ depends on the incomes and expenses received during the system's operation:

$c_{1}^{(k)}$ is the income received for one condition maintenance;

$c_{2}^{(k)}$ is the payment for a time unit of one device's operation during maintenance;

$c_{3}^{(k)}$ is the payment for a time unit of one device being idle;

$c_{4}^{(k)}$ is the payment for a time unit of one condition's stay in queue; and,

$c_{5}^{(k)}$ is the payment for one lost condition of the $k$ type.

The final stage of the study is to construct an optimal management strategy. To solve this problem, we use the well-known fact [15]: if a fractional-linear functional has an extremum (maximum or minimum), then this extremum is achieved in the class of degenerate deterministic strategies.

Concerning the given problem there are two subsystems (two lanes - two types of queries). 
The subsystem of $k$-th type, per designations of Kendall's notation, can be described as follows: $C B S M A P / M_{k} / n_{k} / N_{k}$, where CBSMAP means that the arrival flow is defined as the controlled arrival-flow, as described above; $M_{k}$ means that duration of service in a subsystem has exponential distribution with parameter $\mu_{k}$ (intensity of the journey through "obstacle" in the direction $\mathrm{k}$ ); and $n_{k}$ and $N_{k}$ define the number of channels of service and number of places in the queue, given as $n_{k}$ and $N_{k}$, respectively. In the given case we can consider $n_{i}=1$ (the service channel understood as right-hand and left-hand throughput "regulators" of the repair equipment that carries out the car's admission), and $N=0$, whereby when there is no turn in the system, and there is a "bunker" of saved-up requests, we consider requests that come into a system as soon as it arrives according to the service.

A request can considered as being "complete" once the passing through of the "obstacle", the service channel, is complete. According to the classification of queueing models, this system can be considered to be a controlled semi-Markov queueing model because its evolution is defined by the controlled semi-Markov process.

In this case, Markov moments are those moments when queries are received within a subsystem (permission to journey from the right or left traffic controller).

The following options are available for constructing the management space: only controlling the type of incoming conditions by determining the type of conditions that are accepted into the system, for example, $i$-type conditions; the choice of the query type and the moment of applications' receipt; the choice of query type; the moment of receipt; and the number of applications in the group.

The equality for control set is fair:

$$
\begin{aligned}
U_{(i, \vec{l})} & =E \times[0, \infty) \times \widetilde{M}_{k}=\{(j, u, s), \\
j \in E & =\{1,2, \ldots, N\}, \quad u \in R^{+}=[0, \infty), \\
& s \in \widetilde{M}_{k}=\left\{1, \ldots, M_{k}\right\}
\end{aligned}
$$

This set of controls consists of a final number of half-lines. The probability measure on the set can be determined as a set of probabilities. To arrive at the decision that the batch of i-th (discrete component),

$$
\begin{gathered}
P\left\{u(t)=\frac{j, s}{\xi(t)}=(i, \vec{l})\right\}=p_{(i, \vec{l}),(j, s)}=p_{(i, \vec{l}), j} p^{(j)}(s), p_{(i, \vec{l}),(j, s)} \geq 0, \\
\sum_{(j, s) \in E \times E_{k}} p_{(i, \vec{l}),(j, s)}=1, \quad j \in E=\{1,2, \ldots, N\}, \quad s \in \widetilde{M}_{k}
\end{gathered}
$$

with conditional distributions of continuous component. In the accepted designations, it is possible to determine conditional distribution of components by equality,

$$
\begin{aligned}
& F_{(i, \vec{l})}(j, u, n)=P\{\theta<u / \xi(t)=(i, \vec{l}), \\
& \quad u(t)=(j, n)\}, \\
& \quad j \in E=\{1,2, \ldots, N\}, \quad u \in R^{+}, \quad n \in \widetilde{M}_{k},
\end{aligned}
$$

while the strategy is determined by equalities,

$$
\begin{aligned}
G_{(i, \vec{l})}(j, u, n) & =P\{u(t)=(j, n), \theta<u / \xi(t)=(i, \vec{l})\}= \\
& =p_{(i, \vec{l}), j} p^{(j)}(n) F_{(i, \vec{l})}(j, u, n), \\
j \in E & =\{1,2, \ldots, N\}, \quad u \in R^{+}, \quad n \in\left(1, \ldots, M_{i}\right) .
\end{aligned}
$$

which can be individual "tariffs" (profits and fines) that are different from those of other zones. For example, profit and fines for the lane $i=1$ may be higher than those for the lane $i=2$, as they depend on the traffic and road situation at the site (for example, previously obtained statistical data, such as flow density in that direction). In this way the "fair" implementation of fines and profits can form a principle of jam minimization on the section 
of the road concerned, or else improve the productivity of repairing vehicles.

The final stage of this study is to construct an optimal management strategy. To solve this problem, we use the following well-known fact: if a fractional-linear functional has an extremum (maximum or minimum), then the extremum is reached in the class of degenerate deterministic strategies [15].

The fixed degenerate measure in condition is defined by the equation

$$
\begin{gathered}
G_{(i, \vec{l})}(j, n)=P\{u(t)=(j, n) / \xi(t)=(i, \vec{l})\}=p_{(i, \vec{l}), j} p^{(j)}(n)=1, \\
G_{(i, \vec{l})}(s, m)=P\{u(t)=(s, m) / \xi(t)=(i, \vec{l})\}=p_{(i, \vec{l}), s}=0, \quad(s, m) \neq(j, n)
\end{gathered}
$$

For a discrete component the following equation is used:

$$
F_{(i, \vec{m})}(j, x, n)=\left\{\begin{array}{l}
1, \tau_{(i, \vec{m})(j, n)}<x \\
0, \tau_{(i, \vec{m})(j, n)}>x
\end{array}\right.
$$

Let us note the moment of the conditions' group receipt and the number of conditions in the group when controlling for the selection of the conditions' type.

It is therefore possible to construct a correspondence:

$$
(i, \vec{m}) \rightarrow j_{(i, \vec{m})}^{(0)}, \quad(i, \vec{m}) \rightarrow\left(j_{(i, \vec{m})}^{(0)}, \tau_{(i, \vec{m})}^{(0)}\right), \quad(i, \vec{m}) \rightarrow\left(j_{(i, \vec{m})}^{(0)}, \tau_{(i, \vec{m})}^{(0)}, n_{(i, \vec{m})}^{(0)}\right)
$$

and every time the state is hit, the relevant decision can be made with probability 1 .

The arrival flow can assume two states. The subsystems are of a single channel and there are no places in the queue; that is, $E=\{1,2\}, n_{1}=n_{2}=1, N_{1}=N_{2}=0$. Considering that applications arrive as the batch comprising one query of a certain typethe journey being the moment a car carries out its movement to the lance concerned-then $M_{1}=M_{2}=1$.

In the case under consideration, Markov moments are moments when any type of conditions arrives in the system. In the case of applications of the k-type receipt, the application data is sent for maintenance to the k-type subsystem, and the group from the zero number of applications "arrives" at the remaining subsystems. The states of the queuing system (the states of the controlled semi-Markov process $\xi(t)$ are determined by the vector $\left(i, l_{1}, l_{2}\right)$, where $i-$ is the state of the input stream (the group of conditions of the $\mathrm{i}$ type arrives at the Markov moment), and $l_{k}-$ is the number of conditions in the $k$ type subsystem, $(k=1,2)$.

Transition from a state $\left(i, l_{1}, l_{2}\right)$ to a state $\left(j, l^{\prime}{ }_{1}, l^{\prime}{ }_{2}\right)$ is carried out with positive probability if $l_{k}^{\prime} \leq l_{k}, k \neq j$, that is, in all subsystems except those of the $j$-th type where there is a service of applications that can be presented as process of pure death. The number of applications in these subsystems does not surpass the number of applications in subsystems at the previous moment at which the group was received. We control the type of queries coming into the system, that is we "order" the type of the following application. Considering the probability measure defined above (6):

$$
\begin{gathered}
G_{\left(i, l_{1}, l_{2}\right)}(j)=P\left\{u(t)=j / \xi(t)=\left(i, l_{1}, l_{2}\right)\right\}=p_{\left(i, l_{1}, l_{2}\right), j}, \\
p_{\left(i, l_{1}, l_{2}\right), j} \geq 0, \quad \sum_{j \in E} p_{\left(i, l_{1}, l_{2}\right), j}=1, \quad E=\{1,2\}
\end{gathered}
$$

To optimize the income functional, we use earlier expressions obtained for each $k$ queuing subsystem: $p_{(i, \vec{m}),(j, \vec{l})}^{(k)}, m_{(i, \vec{m})}^{(k)}, s_{(i, \vec{m})}^{(k)}$.

We construct functional accumulation on the trajectories of the controlled semi-Markov process. The income function for the system of the set type is a fractional and linear functional. If the fractional and linear functional has an extremum (a maximum or a 
minimum), then this extremum is reached in a class of the degenerate-determined strategy.

We will define the number of degenerate strategies in the conditions of the problem. Considering that the set of states comprises four states, and that one or another decision can be made within each state (the first or second type of the following group can be chosen), the number of strategy equals 16 .

Take into account all possible states of the system.

$(1,1,0)$ - at the markov moment the first type of query arrives, there is one service query in the first type subsystem, there are no queries in the second type subsystem.

$(1,1,1)$ - the query of the first type arrives at the markov moment, there is one service query in the first type subsystem, there is one query in the second type subsystem.

$(2,0,1)$ - the query of the second type arrives at the markov moment, there are no queries in the first type subsystem, there is one query in the second type subsystem.

$(2,1,1)$ - at the markov moment the query of the second type arrives, there is one query in the first type subsystem, there is one query of the second type.

We provide just a few conclusions as examples of the calculated results due to the large number of computations involved, and also to save space.

For example, at the choice of strategy

the income functional is

$$
\begin{aligned}
& p_{(1,1,0), 1}=1, p_{(1,1,0), 2}=0 \\
& p_{(1,1,1), 1}=1, p_{(1,1,1), 2}=0 \\
& p_{(2,0,1), 1}=1, p_{(2,0,1), 2}=0 \\
& p_{(2,1,1), 1}=1, p_{(2,1,1), 2}=0
\end{aligned}
$$

At the choice of strategy

$$
S=\frac{\left(\begin{array}{l}
\int_{0}^{\infty}\left[c_{1}^{(1)}\left(1-e^{-\mu_{1} t}\right)+c_{2}^{(1)} \frac{1-e^{-\mu_{1} t}}{\mu_{1}}+\right. \\
c_{3}^{(1)}\left[t-\frac{1-e^{-\mu_{1} t}-\mu_{1} t e^{-\mu_{1} t}}{\mu_{1}\left(1-e^{-\mu_{1} t}\right)}\right]\left(1-e^{-\mu_{1} t}\right)+ \\
\left.+c_{5}^{(1)} e^{-\mu_{1} t}\right] d F_{11}(t)
\end{array}\right)}{\int_{0}^{\infty} \bar{F}_{11}(x) d x}
$$

the income functional is

$$
\begin{aligned}
& p_{(1,1,0), 1}=1, p_{(1,1,0), 2}=0 \\
& p_{(1,1,1), 1}=1, p_{(1,1,1), 2}=0 \\
& p_{(2,0,1), 2}=1, p_{(2,0,1), 1}=0 \\
& p_{(2,1,1), 1}=1, p_{(2,1,1), 2}=0
\end{aligned}
$$

$$
S=\frac{\left(\begin{array}{l}
\int_{0}^{\infty}\left(c_{1}^{(2)}\left(1-e^{-\mu_{2} t}\right)+c_{2}^{(2)} \frac{1-e^{-\mu_{2} t}}{\mu_{2}}+c_{3}^{(1)} t+\right. \\
+c_{3}^{(2)}\left[t-\frac{1-e^{-\mu_{2} t}-\mu_{2} t e^{-\mu_{2} t}}{\mu_{2}\left(1-e^{-\mu_{2} t}\right)}\right]\left(1-e^{-\mu_{2} t}\right)+ \\
\left.c_{5}^{(2)} e^{-\mu_{2} t}\right) d F_{22}(t)
\end{array}\right)}{\int_{0}^{\infty} \bar{F}_{22}(x) d x}
$$

To search for a maximum for the fractional and linear function on the set of degenerate distributions, it is necessary to choose a maximum value from 16 values; this is in addition to the strategy corresponding to this value, which will be optimum. Therefore, explicit functions can therefore be provided for the proposed strategies.

\section{Conclusion}

Algorithmization of model and also the main structure is presented. The example of control on the basis of CBSMAP-model is given. The model allows to optimize road traffic in case of road repair works on lanes. 
The income functionality is presented for certain strategies.

The given algorithm makes it possible to make the process of selecting the number of allowed queries optimal (taking into consideration) the specified limits on the income and expenditure of the system. In further studies, it is possible to modify the model given the increase in the number of road lanes.

\section{References}

1. B. Bartosiewicz, Sz. Wiśniewski. Transport Problems, 10(3), 87-99 (2015) DOI: 10.21307/tp-2015-037

2. B. Mrówczyńska. Transport Problems, 10(3), 141-152 (2015) DOI: 10.21307/tp-2015042

3. K. Yang, M. Menendez, N. Zheng. Transportation Research Part C, 105, 439-455 (2019) DOI: $10.1016 /$ j.trc.2019.06.007

4. P. M. Kumar, U. Devi G, G. Manogaran, et al. Computer Networks, 144, (2018) 154 162. DOI: 10.1016/j.comnet.2018.07.001

5. M. Martínez-Díaz, I. Pérez. Transportation Research Part B: Methodological, 75, 19-35 (2015) DOI: 10.1016/j.trb.2015.02.003

6. J. Jin, X. Ma, I. Kosonen. Advances in Engineering Software, 114, 348-360 (2017) DOI: $10.1016 /$ j.advengsoft.2017.08.005

7. T.S.Babicheva, D.S.Babichevb. Procedia Computer Science, 55, 461-468 (2015) DOI: 10.1016/j.procs.2015.07.014

8. S. Salmana, S. Alaswadb. Computers \& Operations Research, 99, 191-205 (2018) DOI: 10.1016/j.cor.2018.06.015

9. L.N.S. Dhulipalaab. M.M. Flint. Reliability Engineering \& System Safety, 193, 106659 (2020). DOI: 10.1016/j.ress.2019.106659

10. W. Bei, C. Lirong, F. Reliability. Engineering \& System Safety, 190, 106516 (2019) DOI: $10.1016 /$ j.ress.2019.106516

11. H. Jin, F. Han, Y. Sangc. Mechanical Systems and Signal Processing, 139, 106570 (2020) DOI: 10.1016/j.ymssp.2019.106570

12. B. Wang, Q. Zhu. Automatica, 94, 72-80 (2018) DOI: 10.1016/j.automatica.2018.04.016

13. R. Castillo González, \& J.B. Clempner, \& A.S. Poznyak. Mathematics and Computers in Simulation (MATCOM), 166(C), 283-297 (2019) DOI: 10.1016/j.matcom.2019.06.002

14. E.V. Kondrashova., V. Kashtanov. 6th International Conference, NAA 2016, 439-447 (2016).

15. V.A. Kashtanov, E.V. Kondrashova. Lecture Notes in Engineering and Computer Science. Proceedings of The World Congress on Engineering 2013, 69-73 (2013)

16. E.V. Kondrashova. International Journal of Mathematical Models and Methods in Applied Sciences, 10, 18-26 (2016)

17. D.M. Lucantoni. Communications in Statistics, Stochastic Models. 7(1), 1-46 (1991) 